\section{THU0239 IDENTIFICATION OF BIOMARKERS AND IMMUNE PATHWAYS FOR PERSONALIZED DRUG TARGETING IN PATIENTS WITH NEWLY DIAGNOSED PRIMARY SJÖGREN'S SYNDROME}

Gwenny M. Verstappen ${ }^{1}$, Lu Gao ${ }^{2}$, Sarah A. Pringle ${ }^{1}$, Janneke H. Terpstra ${ }^{1}$, Silvia C. Liefers ${ }^{1}$, Jolien F. van Nimwegen ${ }^{1}$, Arjan Vissink ${ }^{3}$, Vishal Patel ${ }^{2}$, Aiqing $\mathrm{He}^{2}$, Amy Truong ${ }^{2}$, Sarah $\mathrm{Hu}^{2}$, Ashok Dongre ${ }^{2}$, Arathi Krishnakumar ${ }^{2}$, Stefan Kirov', Julie Carman ${ }^{2}$, Hendrika Bootsma ${ }^{1}$, Frans G.M. Kroese ${ }^{1} .{ }^{1}$ University of Groningen, University Medical Center Groningen, Rheumatology and Clinical Immunology, Groningen, Netherlands; ${ }^{2}$ Bristol-Myers Squibb, New Jersey, United States of America; ${ }^{3}$ University of Groningen, University Medical Center Groningen, Oral and Maxillofacial Surgery, Groningen, Netherlands

Background: Patients with primary Sjögren's syndrome (pSS) present with heterogeneous clinical symptoms and immune dysregulation. How immune dysregulation in SS arises is poorly understood, which hampers development of effective therapies. Identification of key immune pathways contributing to pSS pathogenesis is essential for successful drug development. Systemic treatment of patients early in the disease process will limit irreversible damage to salivary and lacrimal glands, as well as to other affected tissues.

Objectives: To identify early systemic biomarkers and dysregulated immune pathways in newly diagnosed pSS patients by multidimensional immuno-profiling

Methods: We included 40 newly diagnosed pSS patients (39 female;

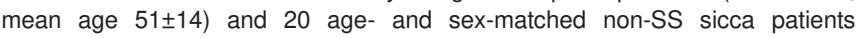
(19 female; mean age $50 \pm 13$ ). All pSS patients fulfilled ACR-EULAR criteria. Serum and peripheral blood mononuclear cells (PBMC) were collected and cryopreserved. PBMCs were thawed for immunophenotyping by flow cytometry and RNA isolation. RNA sequencing was performed using TruSeq Stranded Total RNA Library Prep Gold (Illumina), following manufacturer's recommendations, and RNAseq libraries were sequenced on a HiSeq2500 system. Additionally, serum proteomics and immunoassays for pro-inflammatory cytokines in serum were performed.

Results: Interferon (IFN) type I signaling pathways were at the top of enriched pathways in PBMCs from pSS patients, compared with non-SS sicca controls (adjusted $\mathrm{p}<0.05$ ). Additionally, the gene signature of

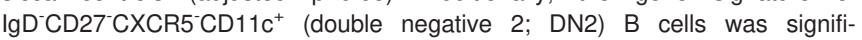
cantly upregulated in pSS patients (adj. $p<0.05$ ). Immunophenotyping analysis showed increased frequencies of $\mathrm{CD}_{4} 0^{+} \mathrm{pDCs} \quad(\mathrm{p}=0.004)$, intermediate monocytes $(p=0.008), \quad \mathrm{ICOS}^{+}$memory $\mathrm{CD}^{+} \mathrm{T}$ cells $(p<0.001)$, cTfh cells $(p=0.001)$, and cTfr cells $(p<0.001)$ in pSS patients. Memory B cells were significantly decreased in pSS patients $(p<0.001)$ Serum proteomics showed that the top proteins up-regulated in pSS patients were immunoglobulin subunits. The top of up-regulated pathways involved IFN signaling (adj. p < 0.05), similar to RNAseq data. Consistently, immunoassays showed increased levels of CXCL10 in serum and MXA in whole blood of pSS patients, compared with non-SS sicca controls $(p=0.009$ and $p=0.001$, respectively). Blood MxA protein levels correlated strongly with the IFN signature score in PBMCs $\left(R s=0.8 ; p=10^{-12}\right)$. The $T$ cell-related biomarkers described above, the frequency of memory $B$ cells, and MXA levels correlated significantly with EULAR Sjögren's disease activity index (ESSDAl) scores, whereas the frequency of $C D 40^{+} \mathrm{pDCs}$, intermediate monocytes, and serum CXCL10 levels did not correlate with these scores. When a correction for multiple testing was applied, only cTfr cells correlated significantly with ESSDAl scores ( $R s=0.5 ; p=0.002$ ).

Conclusion: Newly diagnosed patients with pSS show co-activation of the IFN type I and B cell activation pathways, compared to non-SS sicca controls. At the same time, $\mathrm{CD}^{+} \mathrm{T}$ cell subsets critical for B cell function are activated, which can enhance B cell activation and plasma cell formation. Personalized treatment based on the activity of each pathway in individual patients potentially increases the efficacy of such treatments. Acknowledgement: Part of this work was funded by NIH grant RFA-DE06-004

Disclosure of Interests: Gwenny M. Verstappen: None declared, Lu Gao Employee of: Bristol-Myers Squibb, Sarah A. Pringle: None declared, Janneke H. Terpstra: None declared, Silvia C. Liefers: None declared, Jolien F. van Nimwegen Speakers bureau: Bristol-Myers Squibb, Arjan Vissink: None declared, Vishal Patel Employee of: Bristol-Myers Squibb, Aiqing He Employee of: Bristol-Myers Squibb, Amy Truong Employee of: BristolMyers Squibb, Sarah Hu Employee of: Bristol-Myers Squibb, Ashok Dongre Employee of: Bristol-Myers Squibb, Arathi Krishnakumar Employee of: Bristol-Myers Squibb, Stefan Kirov Employee of: Bristol-Myers Squibb, Julie Carman Employee of: Bristol-Myers Squibb, Hendrika Bootsma Grant/research support from: Unrestricted grants from Bristol-Myers Squibb and Roche, Consultant for: Roche, Bristol-Myers Squibb, Novartis, Medimmune, Union Chimique Belge, Speakers bureau: Bristol-Myers Squibb, Novartis, Frans G.M. Kroese Grant/research support from: Unrestricted grant from Bristol-Myers Squibb, Consultant for: Bristol-Myers Squibb, Speakers bureau: Bristol-Myers Squibb, Roche, Janssen-Cilag DOI: 10.1136/annrheumdis-2019-eular.3148

\section{THU0240 GENE EXPRESSION SIGNATURES ARE RELATED TO SPECIFIC SUBSETS OF PATIENTS WITH SYSTEMIC LUPUS ERYTHEMATOSUS}

M. Javad Wahadat ${ }^{1,2}$, Noortje Groot ${ }^{2}$, C.G. van Helden-Meeuwsen ${ }^{3}$, Iris LA Bodewes ${ }^{3}$, Erika Huijser ${ }^{3}$, Sylvia Kamphuis ${ }^{2}$, Marjan Versnel ${ }^{3} .{ }^{1}$ Erasmus $^{3}$ University Medical Center, Department of Immunology, Rotterdam, Netherlands; ${ }^{2}$ Sophia Children's Hospital, Erasmus University Medical Center, Department of Pediatric Rheumatology, Rotterdam, Netherlands; ${ }^{3}$ Erasmus University Medical Center, Department of Immunology, Rotterdam, Netherlands

Background: Systemic lupus erythematosus (SLE) is a heterogeneous, lifelong autoimmune disease, with a more severe phenotype in children compared to adult-onset SLE [1]. To date, approved drugs are by far not effective enough and have significant side effects, especially due to the use of prednisone. Although profiling of the blood transcriptome revealed a prevalent IFN type I signature in SLE, blocking the IFN pathway only showed efficacy in a subset of adult-onset SLE patients. Recent studies revealed that other gene signatures like IFN type I+II (M5.12), B cell, plasmablast and neutrophil signatures are important in SLE as well and some could be linked to specific clinical phenotypes [2, 3]. Assessment of these novel gene signatures in SLE may help to distinguish specific disease phenotypes and guide treatment choices in the future.

Objectives: To determine and compare the expression of the IFN-, B cell-, plasmablast- and neutrophil signatures in pediatric and adult SLE patients.

Methods: The IFN-I-, M5.12-, neutrophil-, B cell- and plasmablast signatures were measured using real-time quantitative PCR expression on whole blood RNA samples. To identify correlated groups of genes and reduce data complexity, the expression of a selection of genes identified by blood transcriptional profiling was tested and subsequently added to a principle component analysis to obtain a limited set of genes (2-5 per signatures), that reliably represent a specific signature.

These signatures were analyzed in three separate pilot cohorts of healthy controls $(n=12)$, pediatric- $(n=22$; average disease duration $=0.9$ years $)$ and adult SLE patients ( $n=36$; average disease duration $=17.4$ years).

Results: IFN-I signature was significantly higher in SLE patients compared to healthy controls $(p=0.001)$. M5.12 $(p=0.05)$ and the B cell $(p=0.0001)$ signature showed a significant difference between the pediatric and adult cohort while there was no difference between the neutrophil- and plasmablast signatures in the two patient groups. Interestingly, the B cell gene signature, correlated with age $(p=0.0001, r=-0.49)$ and disease duration $(p=0.001, r=-0.42)$. In addition, the possible correlation between the IFNsignature and the other signatures was investigated. While the IFN-I signature in both adults and children showed a significant positive correlation to the M5.12- $(p<0.0001, r=0.97 ; p=0.006, r=0.61)$ and plasmablast $(p=0.03, \quad r=0.37 ; \quad p=0.0037, \quad r=0.62) \quad$ signature expression, only adult patients had a significant positive correlation of the IFN-I signature to the neutrophil signature $(p=0.001, r=0.55)$.

Conclusion: In this pilot study we found significant differences in gene expression signatures between pediatric and adult SLE patients. Additionally, age and disease duration were significantly correlated to the B cell gene signature. These findings indicate differences in transcriptional profiles in specific subsets of SLE patients which could have therapeutic consequences.

\section{REFERENCES:}

[1] Groot, N., et al., Long-term clinical outcomes in a cohort of adults with childhood-onset Systemic Lupus Erythematosus. Arthritis Rheumatol, 2018.

[2] Banchereau, R., et al., Personalized Immunomonitoring Uncovers Molecular Networks that Stratify Lupus Patients. Cell, 2016. 165(3): p. 551-65.

[3] Bodewes, I.L.A., et al., Systemic interferon type I and type II signatures in primary Sjogren's syndrome reveal differences in biological disease activity. Rheumatology (Oxford), 2018. 57(5): p. 921-930.

Disclosure of Interests: M. Javad Wahadat: None declared, Noortje Groot: None declared, C.G. van Helden-Meeuwsen: None declared, Iris LA Bodewes: None declared, Erika Huijser: None declared, Sylvia Kamphuis: None declared, Marjan Versnel Grant/research support from: MAV received financial support from Domainex. DOI: 10.1136/annrheumdis-2019-eular.4659 